\title{
IMPROVING THE TRACKING PERFORMANCE OF MECHANICAL SYSTEMS BY ADAPTIVE EXTENDED FRICTION COMPENSATION
}

\author{
B. De Jager ${ }^{1}$ \\ Department of Mechanical Engineering, Eindhoven University of Technology, P.O. Box 513 \\ 5600 MB Eindhoven, The Netherlands
}

\begin{abstract}
The paper discusses a tracking control system and shows with simulation and experimental results that extended friction models can be successfully incorporated in a computed-torque-like adaptive control scheme. The friction model used includes Coulomb, viscous, and periodic friction with sense of direction dependent parameters. To get small tracking errors, adaptation of the friction model parameters is necessary. The tracking performance is an order of magnitude better than with PD control. The robustness of the scheme for parameter inaccuracies is sufficient, owing to the adaptation, but the controller gains are limited due to stability problems caused by unmodeled dynamics.
\end{abstract}

Key Words. Nonlinear control systems; adaptive control; robots; tracking systems; robustness; friction compensation

\section{INTRODUCTION}

\subsection{Motivation}

The performance of high-speed path-following mechanical systems can be enhanced by using advanced tracking control schemes. In general, the effects of friction, backlesh, hysteresis and other unpleasant real world phenomena are not considered in the control schemes in literature, because these effects cannot easily be handled by the mathematical machinery used to prove, e.g., boundedness, stability or exponential convergence of the tracking error. Often, however, these phenomena establish the limits of performance.

The presence of friction in mechanical systems, where material parts move relative to each other and where contact is necessary due to a guiding or bearing function of the parts, is unavoidable. It is not always possible to eliminate friction by using advanced tribological measures. When traditional techniques to eliminate backlash are used, e.g., pre-tension causing larger forces normal to the contact surface, the problem of friction becomes even more pronounced. In general, friction is a limiting factor for the tracking performance of mechanical control systems.

There are several ways to rectify the effect of friction:

- the use of high-gain feedback; this has disadvantages, such as large input signals and no robust performance due to excitation of high-frequency unmodeled dynamics.

- the use of additional dither signals that prevents the system from stiction and reduces limit cycles; this may lead to fatigue due to high frequency excitation and only assures perfect tracking in the mean, compensation of friction by the controller; the accuracy of the compensation largely depends on the correctness of the structure of the friction model used and on an accurate knowledge of the friction model parameters.

Here, the focus is on friction compensation to overcome the disadvantages of friction. To use it effectively some problems have to be addressed.

\subsection{Friction Modeling}

The main problem is the formulation of accurate friction models, which is necessary because incorrect or incomplete compensation of friction may lead to instabilities (Brandenburg $e t$ al., 1987). These models are difficult to obtain, due to the complexity of friction phenomena. Even the physical causes of friction are not well understood (Armstrong-Hélouvry, 1991; Hzessig and Friedland, 1991). If also the dependence of the Coulomb friction on the normal force at the points of contact is considered the analysis may become complicated (Gogoussis and Donath, 1988).

One approach to obtain those models is to perform some measurements on the system in question and deduce an indication of the structure of the equations describing the effects of friction. Some experiments in this direction are performed by Armstrong (1988). but the conclusions with respect to the structure of the friction model are closely related to the system in vestigated and can hardly be generalized. A systematic method can be used, e.g., a tracking error based sequential off-line identification method proposed by Johnson

\footnotetext{
'Email: jag@wfw.wib.tue.nl F.ax: +31 40447355
} 
and Lorenz (1992), that is a bit elaborate and has to be repeated for each system to be controlled.

Another approach is to use an elaborate friction model, and to adapt the parameters of the model. When some terms in the model are not significant, the corresponding parameters will be small. After an initial period of use, the structure of the friction model can be simplified by deleting terms that are related with small parameters, i.e., insignificant terms, or have parameters of equal value, e.g., for direction dependent parameters. It is necessary to use a sufficiently rich model to encompass all relevant effects that can appear and are related to friction. Yet, the number of parameters should not be too large, to avoid problems with the adaptation (overparametrization) and to avoid modeling of disturbances that are not related to friction. The advantage of this approach is that it should be independent of the specific system to be controlled.

Still another approach, chosen in this work, is to investigate the structure of the friction model by using friction compensation with several friction models and adapt the model parameters. Then, looking at the tracking accuracy when the friction parameters are sufficiently adapted, decide if an additional term in the friction compensation does improve it. If not, this term is not appropriate and can be discarded.

\subsection{Previous Work}

Adaptive friction compensation has been used previously (Canudas et al., 1987; Canudas de Wit et al., 1991; Niemeyer and Slotine, 1991; Friedland and Park. 1992), but they use relatively simple friction models. Yang and Chu (1993) include a discussion of the validation of a friction model, assuming a correct model structure so both measurement and process noise can be regarded as resulting from white noise processes. Baril and Gutman (1991) use an adaptive scheme that is specifically targeted at adaptation of the friction parameters. To counteract uncertainty in other parts of the model a robust controller is used.

It is also possible to compensate friction by estimating the friction force with a nonlinear observer (Maron, 1989). Other control schemes use repetitive control (Tung et al., 1993) or table lookup methods (Armstrong, 1988). The first is anly suitable for repetitive tasks. The last is hampered by the fact that to get good results the table has to be set up for each system and has to be updated regularly because the friction changes in time due to aging, wear, maintenance, etc.

Friction compensation is not only used in tracking control. but also in hybrid force/position control to counteract the friction between the manipulator and the environment (Bona and Indri. 1993).

\subsection{Present Work}

The main contribution of this paper is the proof of the viability of the use of a more-elaborate friction model then generally used. A discussion of the robustness of the parameter estimates and of the obtainable tracking error, compared with a PD controller and with simple friction compensation, is also included.
The paper differs in several aspects from most of the other papers discussing adaptive friction compensstion. First, the friction model is more involved, including Coulomb, viscous, and periodic friction components. Although periodic friction has been recognized in experimental data by several authors (ArmstrongHélouvry, 1991; Canudas de Wit et al., 1991), none of them tried to compensate it adaptively. Second, the parameter adaptation is embedded in a general computedtorque-like adaptive control scheme that addresses the adaptation of other model parameters, e.g., the inertia parameters to accommodate load changes, also, and can be used for nonlinear mechanical systems. This makes a uniform treatment of all unknown parameters possible. Further on, in our setup no specific friction model has to be chosen in advance, only a class or sequence of friction models.

The proof of viability is given in the following order. First, Section 2 discusses the experimental system, a model of the system, the friction model, and the adaptive control scheme used. Then, in Section 3, the setup for the numerical and real-world experiments is given. Sections 4 and 5 present the simulation and experimental results. The discussion of the results follows in Section 6. Finally, Section 7 shows the conclusions and recommendations.

\section{SYSTEM, MODELS, AND CONTROLLER}

This section presents the experimental system, a simple model of this system, and the friction model used. including some background why this type of model is chosen. The section closes with the presentation of the control scheme used in the simulations and experiments to control the model and the experimental system, respectively.

\subsection{Experimental System}

The system used for the experiments is a two (or three) degrees-of-freedom manipulator, moving in the horizontal plane, with three prismatic joints, two of which are parallel and coupled by a spindle with adjustable flexibility. It is a so-called TT-robot or, emphasizing the Cartesian coordinates, an XY-table. For a schematic drawing of the XY-table, see Fig. 1.

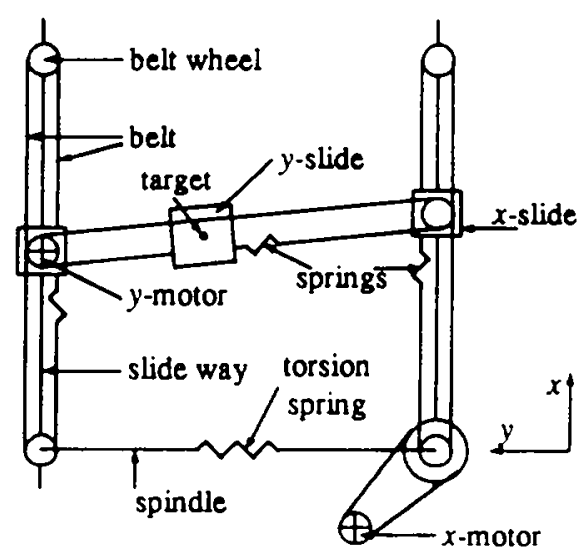

Fig. 1. Schematic drawing of XY-table 
The main characteristics of the system are

- working area $1 \times 1[\mathrm{~m}]$,

- two permanent magnet DC motors,

- two current amplifiers.

- optical encoders for the motor positions.

- microcomputer based control,

- adjustable dynamics since the torsion spring in the spindle can be replaced easily, so the spindle stiffness can be adjusted.

The adjustable torsion spring in the spindle, and the springs connecting the belts with the $x$-slides and $y$ slide, can be used to study the robustness of control schemes and to evaluate controllers for systems with flexible joints. For the results published here, the stiffest spring in the spindle was used, so only marginal unmodeled dynamics was introduced by it.

The motors are connected by belts and pre-loaded springs with the slides. These springs are not very stiff and are a main limitation for the use of high bandwidth controllers. For the $x$-motor an additional transmission with a ratio of $60 / 13$ is used to adapt the motor characteristics. This also leads to a higher resolution of the position measurement, because the code-wheels are mounted on the motor shafts. The motor currents are controlled by current amplifiers, whose setpoints are generated by the control system.

The main task of the system is to let a target point, the center of the $y$-slide or end-effector, follow a desired trajectory. Because the end-effector position is not measured, the derived task is to let the motor positions follow a corresponding trajectory, that is computed based on a stiff model of the XY-table.

\subsection{System Model}

Before giving a model of the XY-table introduce a fairly general multi-body model of a mechanical system

$$
M(q, \vartheta) \ddot{q}+C(q, \dot{q}, \vartheta) \dot{q}+g(q, q, \vartheta)=f .
$$

Here, $M(q, \vartheta)$ is the $n \times n$ positive definite inertia matrix with model parameters $\vartheta . C(q, q, \vartheta) q$ is the $n$ vector of Coriolis and centripetal forces, $g(q, \dot{q}, \vartheta)$ the $n$ vector of gravitational forces. Coulomb and viscous friction. and other state dependent forces, $f$ the $n$ voctor of generalized control forces (forces or torques). In this model each of the $n$ degrees-of-freedom $q$ has its own motor. The dynamics of the motors, sensors, and current amplifiers, backlash, and flexibility of the joints and links are neglected.

The equations for a simple model of the XY-table of Fig. 1, assuming stiff joints and links, are

$$
\begin{aligned}
& \vartheta_{1} \ddot{x}+g_{x}(x, \dot{x})=f_{x} \\
& \vartheta_{2} \dot{y}+g_{y}(y, \dot{y})=f_{y}
\end{aligned}
$$

where $x$ and $y$ are the two prismatic degrees-of-freedom (the components of $q$ ), $f_{x}$ and $f_{y}$ are the control forces in $x$ and $y$ direction, $\vartheta_{1}$ and $\vartheta_{2}$ are the inertia parameters in $x$ and $y$ direction. The functions $g_{x}$ and $g_{y}$ represent disturbance forces due to Coulomb, viscous, and other types of friction or due to other state dependent disturbances. These functions depend on some parameters also and will be detailed further in the next section. Coriolis and centripetal forces are neglected, because there is almost no coupling between movements in $x$ and $y$ direction. Gravitational forces are absent because the manipulator moves in the horizontal plane. The absence of these forces makes the XY-table an ideal object for the study of the merits of friction compensation.

\subsection{Friction Model}

Friction phenomena can by listed in the following (not exhaustive) categaries (Canudas de Wit et al., 1991)

- Coulomb friction and stiction: $a_{c} \operatorname{sgn} q$.

- viscous friction: $a_{v} q$.

- periodic friction: $b_{p} \sin \left(\omega_{p} q+\varphi_{p}\right)$.

- downward bends in the friction force-speed characteristic at low velocities.

The friction may be sense of direction dependent also.

Low-velocity friction phenomena are not very relevant here, because the reference trajectory requires high speeds. Coulomb and viscous friction are often present in mechanical systems. For the XY-table their presence was verified by some simple experiments. By stepping through a sequence of torque commands (setting the setpoint of the current amplifiers) and by computing the corresponding mean steady-state velocity from the position measurements, the characteristics of Coulomb and viscous friction were revealed.

Previous experiments performed to assess the robustness of adaptive control schemes (de Jager, 1992), are used to guide the selection of other components for a favorable friction model. In these experiments it appears that the tracking error in $y$-direction is larger than the error in $x$-direction. Also, it can be deduced from the characteristics of the tracking error in $y$-direction, that there is a harmonic disturbance force. which attributes to the lower tracking accuracy. The periodic force cin be recognized by moving the end-effector by hand. A periodic variation of the force required to move the end-effector with constant speed is easily perceptible.

The origin of the disturbance force can be deduced from its harmonic nature. The period of the force fluctuation is equivalent to the time needed for one complete revolution of the $y$-motor, and so of its shaft, bearings, and belt wheel. Therefore, it seems logical to assume that the disturbance force stems from some imperfections and friction in the shaft and bearings. Other possible explanations could be brush friction, or the presence of imperfections in the electro-magnetic fields in the motor due to, e.g., a lack of rotational symmetry, leading to an inhomogeneous magnetical or electrical field. If the motor is brush-less, the motor constant (relating motor current to torque) is position dependent (Hori and Uchida, 1990), so current control cannot be identified with torque control and the result is a periodic torque variation for constant current and velocity. Then state dependent disturbances are modeled and not friction per se. The use of a reduction in the transmission for the $x$-motor alleviates these motor-bounded effects for the $x$-direction. 
A solution for the periodic friction would be to eliminate it by replacing the shaft and bearings, but, incidentally, it provides a source of model error that does not endanger the stability, but significantly reduces the performance. Almost none of the control schemes proposed in the literature can cope directly with this type of disturbance, except by using larger gains in the PD part of the schemes, but those large gains do endanger the stability and can therefore not be applied in practice.

Another solution is canceling the disturbance force by compensation. This can be regarded as an extension of standard Coulomb friction compensation; it just requires an extended friction model.

The appearance of periodic or position-dependent friction components has been observed previously (Armstrong-Hélouvry, 1991). There position-dependent friction was positively identified by using Fisher statistics, but for their system this friction component was small, in the order of $7 \%$ of the Coulomb friction. In our case this is not true, so periodic friction should be considered explicitly.

When the compensation is based on the angular position $\omega_{p} q$ of the motor shaft, where $\omega_{p}$ is the spatial frequency, only the amplitude $b_{p}$ and phase $\varphi_{p}$ of the sinusoidal compensation force

$$
f_{p}=b_{p} \sin \left(\omega_{p} q+\varphi_{p}\right)
$$

has to be determined. Here $q$ can be interpreted as one component of the degrees-of-freedom, and then the parameters $b_{p}, a_{p}$, and $\varphi_{p}$ are scalar, or it can be the complete column of degrees-of-freedom as in (1), and then these parameters are (diagonal) matrices. It is not necessary to make an explicit distinction between these two cases.

When adaptive controllers are used, one could try to use adaptive friction compensation by estimating amplitude and phase. But, when the compensating force is of the form (3) the parameter $\varphi_{p}$ does not appear linear in the control force, which is required for the adaptation part of the controller to be used. The spatial frequency $\omega_{p}$ is assumed to be known exactly to avoid the same problem. For the XY-table this is not a severe limitation since $\omega_{p}$ depends only on geometric properties, i.e., the diameter of the belt wheel. that can be determined accurately. Fiddling with the phase $\varphi_{p}$ to get a small error is possible, but tedious and should be repeated for each arrangement of belt wheels and belt, and must be repeated every time the connection between motor, belt wheel and end-effector is changed, and after each maintenance. So, a much better solution is to incorporate the adaptation of the phase in the control scheme. For this purpose (3) is written as

$$
f_{p}=a_{p_{1}} \sin \left(\omega_{p} q\right)+a_{p_{2}} \cos \left(\omega_{p} q\right) .
$$

Now the two amplitudes $a_{p_{1}}=b_{p} \cos \left(\varphi_{p}\right)$ and $a_{p_{2}}=$ $b_{p} \sin \left(\Phi_{p}\right)$ and no phase has to be adapted. Both parameters appear linear in the control force. A disadvantage of this method is that both sine and cosine have to be computed, resulting in a longer computation time.

So, including Coulomb, viscous, and periodic friction in the model, the following expression for the friction force $g^{+}$, for $q \geq 0$, and $g^{-}$. for $q<0$, is obtained

$$
\begin{aligned}
& g^{+}(q, q)=a_{c}^{+}+a_{v}^{+} \dot{q}+a_{p_{1}}^{+} \sin \left(\omega_{p} q\right)+a_{p_{2}}^{+} \cos \left(\omega_{p} q\right) \\
& g^{-}(q, q)=-a_{c}^{-}+a_{v}^{-} \dot{q}+a_{p_{1}}^{-} \sin \left(\omega_{p} q\right)+a_{p_{2}}^{-} \cos \left(\omega_{p} q\right)
\end{aligned}
$$

where it is assumed that all parameters in the friction model depend on the sign of the velocity, so there is no need to multiply the harmonic terms with sgn $q$ explicitly. This is also true for the Coulomb friction, or $a_{c}$, terms. The determination of the Coulomb friction for $q=0$ is more involved than described by (4), see the Appendix.

For model (4), that has been deduced from experiments, the possibility that some of its terms are not relevant and can be dropped is not expected. The model is valid. Still, it may be incomplete.

\subsection{Adaptive Controller}

The control scheme used is the passivity based adaptive controller proposed by Slotine and $\mathrm{Li}$ (1988). See also the comments in Spong et al. (1990). This scheme has an approximate feedforward component, based on an estimate of the manipulator dynamics and a virtual reference trajectory, and has a PD component. The generalized control force is just the sum of these components

$$
f=\hat{M}(q) \ddot{q}_{r}+\hat{C}(q, \dot{q}) \dot{q}_{r}+\hat{g}(q, \dot{q})+K_{\nu} s
$$

where $\hat{M}=M(q, \hat{\vartheta}), \hat{C}=C(q, \dot{q}, \hat{\vartheta})$, and $\hat{g}=g(q, q, \hat{\vartheta})$ are the same as the corresponding terms in (1), with $\hat{\vartheta}$ an estimate of the model parameters $\vartheta, q_{r}=q_{d}+\Lambda q$ a virtual reference trajectory, $s=\bar{q}+\Lambda \bar{q}$ a measure of tracking accuracy, $\bar{q}=q_{d}-q$ the tracking error, and $q_{d}(t), \dot{q}_{d}(t), \ddot{q}_{d}(t)$ the desired trajectory. Due to the definition of $s$, the term $K_{v} s$ represents a proportional and derivative action.

The reasons for using derivatives of $q_{r}$ instead of $q_{d}$ in (5) are that the addition of the term $\Lambda \tilde{q}$ in $\dot{q}_{r}=q_{d}+\Lambda \grave{q}$ is a sufficient modification to enable the completion of the stability proof of the control scheme. It also implicitly increases the proportional and derivative action. A disadvantage of this term is that it may cause parameter drift in the presence of measurement noise on $q$ when the system is not sufficiently excited. For a thorough discussion and other suitable modifications see Berghuis (1993). An experimental evaluation of a range of similar control schemes is given by Whitcomb et al. (1993).

Adaptation of the model parameters used in $\hat{M}, \hat{C}$, and $\hat{g}$ is based on the reasonable assumption that, with an appropriate choice of parameters, the generalized control force (5) is linear in the parameters $\hat{\vartheta}$ and can be expressed as

$$
f=Y\left(q, \dot{q}, \dot{q}_{r}, \ddot{q}_{r}\right) \hat{\vartheta}+K_{v} s .
$$

Especially when intricate friction models, e.g., for low velocities, are used this "linear in the parameters" property does not hold and an approximation of the friction function is necessary (Canudas de Wit et al., 1991). 
The adaptation proceeds according to

$$
\hat{\vartheta}=\Gamma^{-1} Y^{T}\left(q, \dot{q}, \dot{q}_{r}, \not{q}_{r}\right) s .
$$

When the initial estimates are chosen as $\hat{\vartheta}\left(t_{0}\right)=0$ and the sdaptation gain $\Gamma^{-1}=0$, the controller of Slotine and $\mathrm{Li}$ becomes a PD controller acting on the tracking error $\not$. When the initial estimates are chosen as $\hat{\vartheta}\left(t_{0}\right)=$ $\vartheta$ and the adaptation gain is 0, a computed-torquelike control scheme without adaptation is obtained. To obtain the "standard" computed torque controller, $q_{d}$ has to be used instead of $q_{r}$ and $q$ in (5).

This control scheme is applied to the model (2) of the XY-table. To rewrite (2) as (1), define the following quantities

$$
\begin{aligned}
& q=\left[\begin{array}{l}
x \\
y
\end{array}\right], \\
& M(q, \vartheta)=\left[\begin{array}{cc}
\vartheta_{1} & 0 \\
0 & \vartheta_{2}
\end{array}\right] \text {, } \\
& C(q, q, \vartheta)=\left[\begin{array}{ll}
0 & 0 \\
0 & 0
\end{array}\right] \text {, }
\end{aligned}
$$

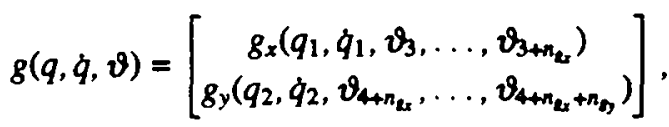

$$
\begin{aligned}
& f=\left[\begin{array}{l}
f_{x} \\
f_{y}
\end{array}\right] .
\end{aligned}
$$

Here, the parameters $\vartheta_{i}, i>2$, correspond in an obvious way to the parameters $a^{+}, a^{-}$in (4), and $n_{8 z}, n_{8 y}$ are the number of friction parameters in $x$ and $y$ direction, respectively. The total number of parameters to be adapted is 18, namely 2 inertia parameters, 8 friction parameters for positive velocities, and 8 friction parameters for negative velocities.

This results in expressions for $Y$ in (6) as follows

$$
\begin{aligned}
& Y^{+}=
\end{aligned}
$$

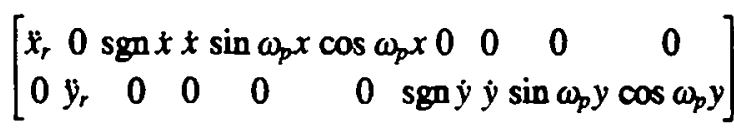

for positive velocities, used for adaptation of the $\vartheta^{+}$ parameters and an equivalent expression $Y^{-}$for negative velocities to adapt the $\vartheta^{-}$parameters, with $\vartheta^{+}=$ $\left[\begin{array}{llll}\vartheta_{1} & \vartheta_{2} & a_{x}^{+} & a_{y}^{+}\end{array}\right]^{T^{2}}$ and $\vartheta^{-}=\left[\begin{array}{llll}\vartheta_{1} & \vartheta_{2} & a_{x}^{-} & a_{y}^{-}\end{array}\right]^{T}$. Of course, because the velocities for $x$ and $y$ direction change sign independent of each other, the updated parameters $a_{x}$ and $a_{y}$ can be selected from both $\vartheta^{+}$and $\vartheta^{-}$at each time instance.

\section{SIMULATION AND EXPERIMENTAL SETUP}

In this section specific information on the setup for the simulations and experiments is given to enable the reader to verify the results given in the next sections. First the control task is discussed, followed by implementation details and the controller design.

\subsection{Control Task}

The control task is to follow a periodic trajectory in the XY-plane, with position control in both coordinate directions. The desired trajectory in Cartesian endeffector space is

$$
\left[\begin{array}{l}
x_{d}(t) \\
y_{d}(t)
\end{array}\right]=\left[\begin{array}{c}
a-R_{d} \cos \psi_{d} \\
b-R_{d} \cos \left(\psi_{d}+\psi_{0}\right)
\end{array}\right]
$$

where $R_{d}=0.2[\mathrm{~m}]$ is the "radius" of the trajectory, $\psi_{d}=\omega_{d} t$, with $\omega_{d}=4 \pi / 3.5[\mathrm{rad} / \mathrm{s}]$, is the desired angular position, and $a=0.8[\mathrm{~m}], b=0.8[\mathrm{~m}]$ specify the center of the working area of the manipulator, see Fig. 2. The constant angle $\psi_{0}$ is used to select the trajectory: if $\psi_{0}=\pi / 2$ the trajectory is a circle, if $\psi_{0}$ has another value the circle is deformed to an ellipse or even a straight (diagonal) line.

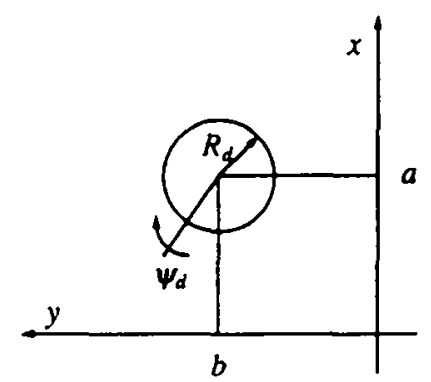

Fig. 2. Desired trajectory

The periodic nature of the task makes it easy to compute accurate and repeatable tracking error statistics. without influence of initial transients. The trajectory has smooth derivatives and generates a range of velocities that makes none of the terms in (4) dominant for the total trajectory.

\subsection{Implementation}

The continuous-time adaptive controller is implemented in discrete time without a modification that compensates for the discrete implementation. The Euler method is used for the integration of the adaptation differential equation (7). Because only the position is measured (by code-wheels) the velocity is estimated. The position is also filtered to diminish the effects of quantization. The position and velocity are predicted one step ahead by a discrete time Kalman filter to compensate for the time delay incurred by the controller computations.

The simulation model of the XY-table is almost implemented as a plug-in-replacement for the experimental system and it includes effects like torque ripple and quantization roundoff not included in the XY-table design model (2). Controllers developed for the simulation model can therefore directly be used in the control system of the XY-table, without the need for an additional translation step between different software implementations, e.g., scaling of measurements and control signals. The Appendix contains a detailed description of the simulation model and its parameters to facilitate reproduction of the simulation results. 


\subsection{Controller Design}

The design of the control parameters $K_{v}$ and $\Lambda$ is performed by choosing a favorable dynamics of the tracking error. characterized by the undamped characteristic frequency $\omega_{c}$ and damping coefficient $\beta_{c}$ of a second order system. These design parameters are related to the control parameters by

$$
\begin{aligned}
\Lambda K_{v} & =\omega_{c}^{2} M \\
K_{v} & =2 \beta_{c} \omega_{c} M .
\end{aligned}
$$

The goal was to get a small tracking error without exciting high-frequency dynamics that could endanger stability.

The selection of $\Gamma^{-1}$ was guided by the rule given in Niemeyer and Slotine (1991), but the gains had to be detuned to avoid stability problems. No extensive tuning of these parameters has taken place.

For the nominal parameter values used for the controller design see Table 1. For the nominal design model parameters, i.e., the inertia parameters in $M$, see Table 2 in the Appendix.

Table 1 Design parameters of the controller

\begin{tabular}{cccl}
\hline Parameter & Value $x$ & Value $y$ & Unit \\
\hline$\omega_{c}$ & $4 \cdot 2 \pi$ & $4 \cdot 2 \pi$ & rad s$^{-1}$ \\
$\beta_{c}$ & 0.7 & 0.7 & - \\
\hline
\end{tabular}

\section{SIMULATION RESULTS}

An overview of the simulation results for extended friction compensation is given. Five sets of results are presented, all for the second of two cycli of 3.5 [s] duration each. Each cyclus contains two circles because $\omega_{d}=4 \pi / 3.5[\mathrm{rad} / \mathrm{s}]$. The results can be divided in two groups. Consideration of Figs. 3-5 indicates the effect of using more-elaborate friction models. Figures 5-7 give an opportunity to assess the effect of using adaptation of parameters instead of fixed parameters in the scheme of Slotine and Li. Now the five sets of results are presented in more detail, but only for the $y$-direction, starting with an assessment of the effects of extended friction compensation.

First, the results without extended friction compensation in $y$-direction are shown. Only inertial forces and the standard Coulomb friction are present in the computed torque part. See Fig. 3. Results without and with adaptation of the parameters are shown. both starting with the nominal parameters. The tracking error is mainly due to the lack of viscous friction compensation. The tracking error is reduced by the adaptation. i.e., the inertia and Coulomb friction parameters are given values, that may change in time, to compensate somehow the effects of the viscous and the periodic friction. Here the Coulomb friction parameter is estimated too high to compensate for the lack of viscous friction compensation. This can be seen in Fig. 3, where the sections of the plots with the largest errors for the upper plot are similar.
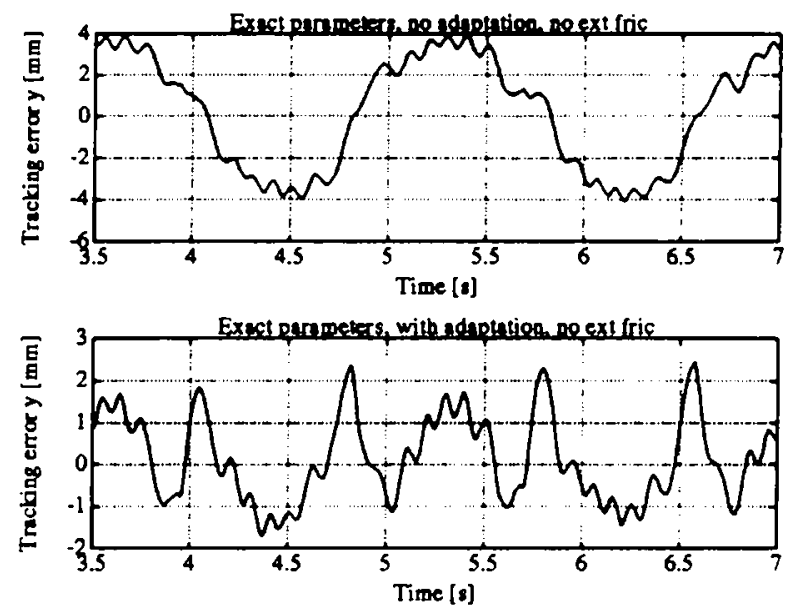

Fig. 3. Simulation results without extended friction compensation

Second, the results without periodic friction compensation in y-direction are shown. Only Coulomb and viscous friction are compensated. Both the results without and with adaptation of the parameters are presented in Fig. 4, starting with the nominal parameters. For the first plot the tracking error is smaller by a factor of 2 . due to the compensation of the viscous friction. Again. the use of adaptation can partly compensate for the unmodeled periodic friction.
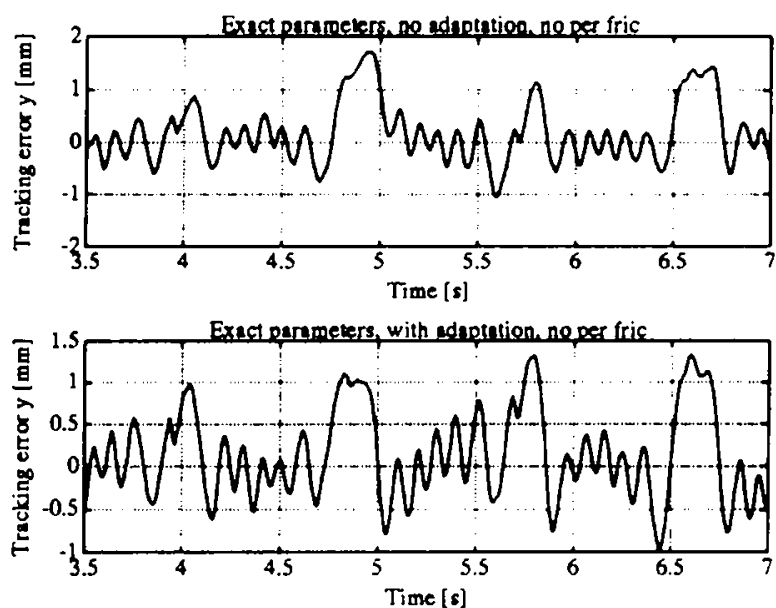

Fig. 4. Simulation results without periodic friction compensation

Third, the results with extended friction compensation in $y$-direction, including Coulomb, viscous, and periodic friction compensation, are considered. The almost ideal tracking error is given in Fig. 5. The results without and with adaptation of the parameters are presented. both starting with the nominal parameters. The remaining tracking error is almost completely caused by the torque ripple. When the torque ripple is absent the error is much smaller, but not equal to 0 due to

1. the sampled data implementation of the controller.

2. the quantization error in the position measurement,

3. the prediction error in position and velocity of the one step ahead Kalman filter.

4. inexact cancellation of the Coulomb friction: the compensation can detect the instance of a change 
of sign of the velocity with an accuracy of 1 sample only, due to the discrete time implementation of the controller, interpolation of the velocity is of no help. because the estimated velocity is too inaccurate.
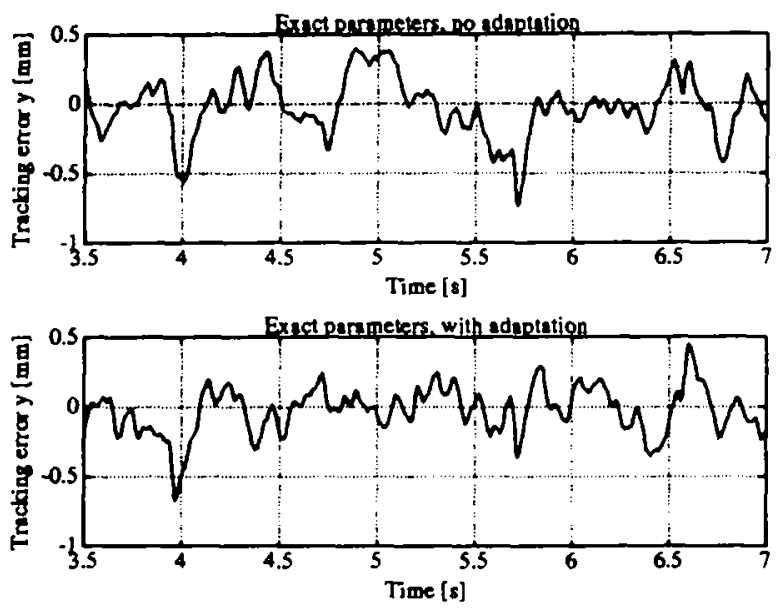

Fig. 5. Simulation results with extended friction compensation

Comparison with the previous figure shows that the addition of periodic friction compensation results in a small, but noticeable, improvement in the performance. In relative terms, it is again a factor of 2 . With adaptation the tracking error is only slightly smaller then without, which means that the parameter adaptation somehow cancels the effects of the four additional causes for the remaining tracking error mentioned above, although the quantization error is mainly of a random nature. Further improvement is hardly possible. due to the lack of structure in the pseudo white noise signal used to model the torque ripple.

To show the influence of the initial parameter estimates and the rate of convergence of the adapted parameters, or better: the rate of convergence of the tracking error due to the adaptation of the parameters, the results starting from an initial parameter estimate of $80 \%$ and $0 \%$ of all nominal parameters are presented in Figs. 6 and 7. So, all parameters, including the inertia and Coulomb friction parameters, are assumed to be approximately known or even completely unknown.

Figure 6 shows the advantage of using adaptation. The tracking error is reduced by a factor of 4 . The adaptation is fast, so an error comparable with the result given in Fig. 5 for exactly known parameters can be obtained after approximately one control cycle.

Finally, the results starting from a zero initial estimate for all parameters clearly show the advantage of using a computed-torque-like control scheme. With a zero initial estimate for the parameters the control scheme of Slotine and Li degenerates to a pure PD feedback of the tracking error. The tracking error is an order of magnitude larger than the error obtainable with more advanced control schemes. Figure 7 also clearly shows that the parameters obtain values that reduce the tracking error significantly after 2 cycli when adaptation is used. Ultimately, the error will be as small as in Fig. 5.
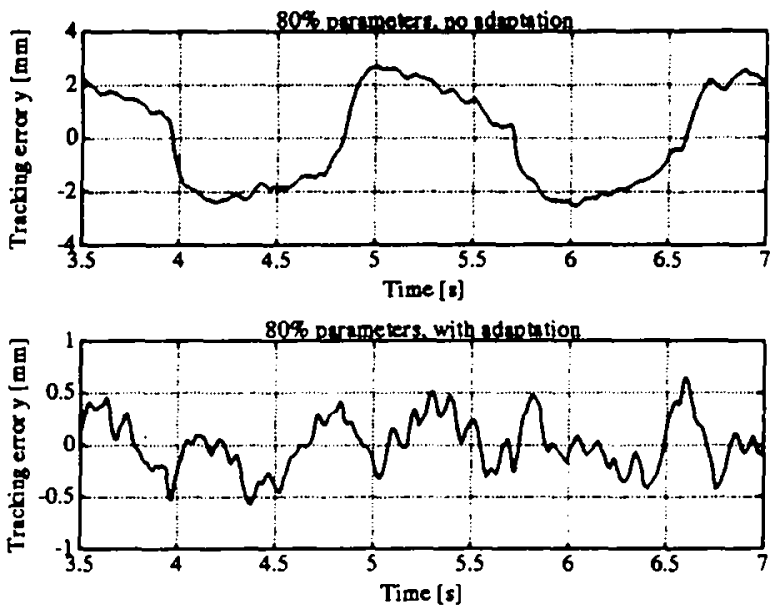

Fig. 6. Simulation results with extended friction compensation, approximate parameters
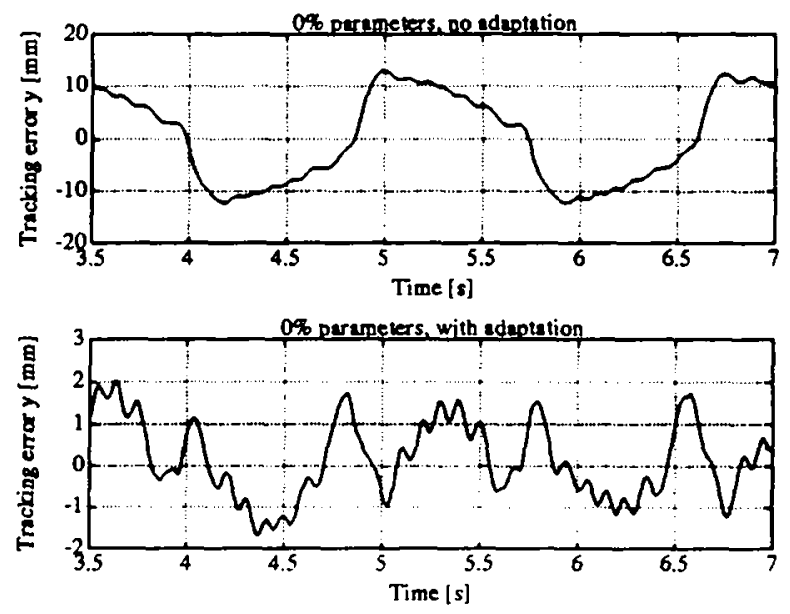

Fig. 7. Simulation results with extended friction compensation, unknown parameters

\section{EXPERIMENTAL RESULTS}

This section presents five experimental results, \&lso for the second of two cycli of 3.5 [s] duration each, except for the last result. The results for PD feedback are obtained by using the controller with zero values of the parameters and no adaptation. From all other experiments only results with adaptation are shown and the initial values of the parameters are assumed to be completely unknown. Results with fixed parameters are not obtained. because, contrary to the simulation, exact model parameters are not defined. The results are presented in order of increasing tracking performance.

A reference result for the tracking error in $y$-direction. presented in Fig. 8, is obtained with a PD controller. Compare this with the first plot in Fig. 7 to see the similarity of simulation and experiment. The tracking error is large. This is due to the low bandwidth of the controlled system $(8[\mathrm{~Hz}])$ relative to the frequency of the desired trajectory $(2 / 3.5[\mathrm{~Hz}])$. Due to the low bandwidth the static controller gain is small also, so low frequency disturbances at the input of the plant. like the Coulomb friction, are not rejected well. An approximate computation using the proportional gain $\Lambda K_{v}=4.3 \cdot(4 \cdot 2 \pi)^{2}=2716$ for the $y$-direction shows that the error due to the lack of friction compensa- 
tion is maximal $8.5[\mathrm{~mm}]$ for the specified trajectory (8). Using acceleration feedforward an improvement of the maximal error with $4[\mathrm{~mm}]$ is possible. Without changes to the controller structure, i.e., acceleration feedforward and compensation of the friction. the tracking error can be reduced by larger feedback gains only. This has been tried, but already a slight increase of the gains caused unacceptable excursions of the input signal.

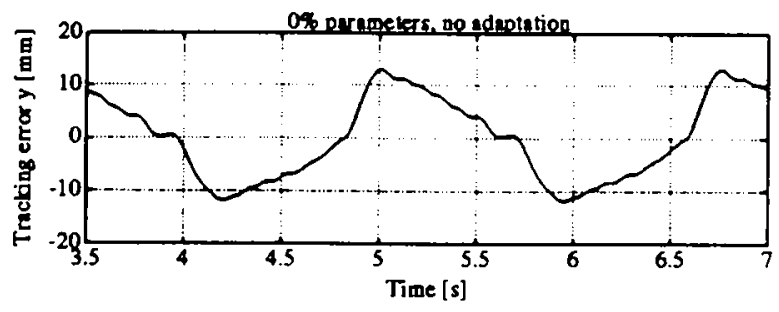

Fig. 8. Experimental result with PD control

Figure 9 gives the result when only inertial forces and Coulomb friction, without sense of direction dependent parameters, are compensated. The error is already small after two cycli, so the adaptation gains are adequate for reasonably fast parameter convergence.

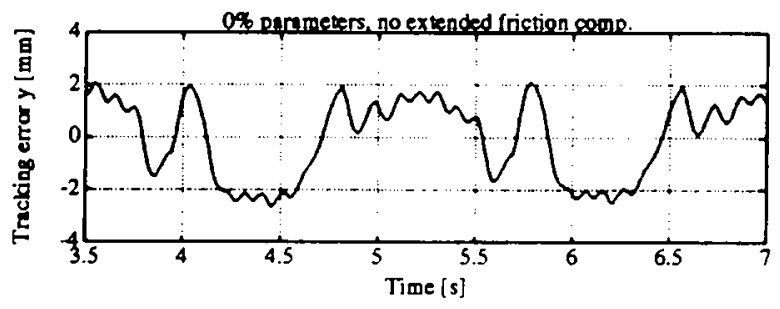

Fig. 9. Experimental result without extended friction compensation, unknown parameters

In Fig. 10 only periodic friction is not compensated. The influence of the sense of direction dependency of the Coulomb friction gives the largest improvement of the tracking error. The effect of the viscous friction compensation is not very large because in Fig. 9 inexact values for the Coulomb friction coefficient already partly compensate for it, as discussed earlier in Section 4 .

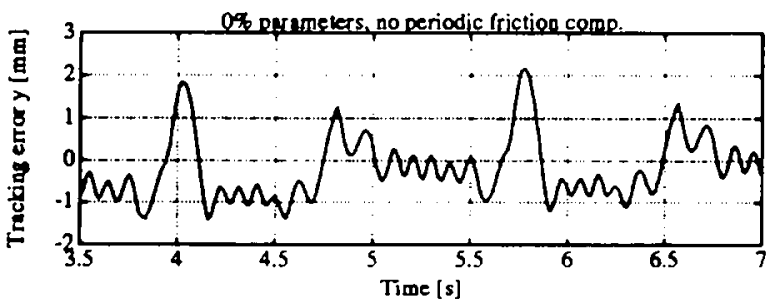

Fig. 10. Experimental result without periodic friction compensation, unknown parameters

Good results are obtained with the full friction model, as shown in Fig. 11. See the second plot of Fig. 7 for comparison with the simulation. This is a rare case where the experimental result is better than the simulation result, but the simulation result is exactly repeatable and the experimental result only within a margin of $\sim 10 \%$ of the tracking error. Also, by using a longer period for the parameter adaptation a further improvement of the tracking error for the simulation has been observed. Compare with the second plot in Figs. 5 or 6 where a smaller tracking error is obtained.

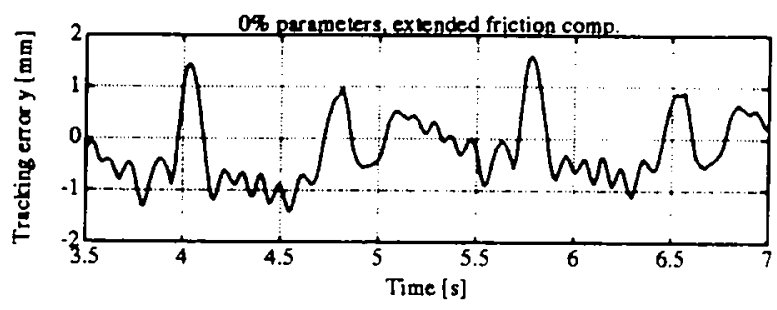

Fig. 11. Experimental result with extended friction compensation, unknown parameters

To unfold the potential of extended friction compensation: the result of Fig. 12, where a longer period to obtain appropriate values $\vartheta$ for the parameters was allowed ( 5 cycli), is the best that could be obtained experimentally, although the improvement is not as large as suggested by the simulation result in the second plot of Fig. 5.

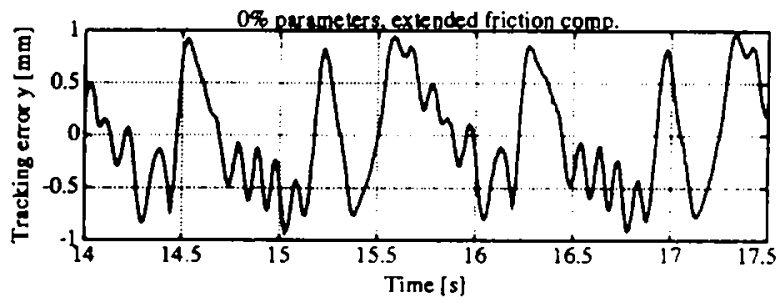

Fig. 12. Experimental result with extended friction compensation, unknown parameters. prolonged adaptation time

It is stressed that to obtain this result both adaptation and an extended friction model are necessary. The periodic components in the tracking error are remnants of the periodic friction in the system that is not completely compensated or is over compensated. A faster adaptation, by choosing larger gains in $\Gamma^{-1}$, was not possible due to stability problems, but the assumption that the parameters are initially completely unknown is also not very realistic. In general the parameters will "converge" within $\sim 7$ [s].

\section{DISCUSSION OF RESULTS}

Both simulations and experiments show a marked performance improvement using the adaptive computed torque scheme with extended friction compensation instead of PD feedback. More extensions of the friction model lead to better performance. When only model based compensation with fixed, but inaccurate, parameters is used, the performance is worse, so adaptation is profitable. Due to stability problems during the experiments, that show up in unacceptable excursions of the control signal, the adaptation could not be tuned to guarantee a "converged" tracking error within 3.5 [s] This was caused by the unmodeled dynamics, that also limit the gains of the PD part of the control scheme. 
To gain more than one order of magnitude in performance. compared with PD feedback, extended model based friction compensation is not sufficient. Compare Figs. 12 and 8, showing an improvement of a factor 12 in tracking performance by taking this measure. A further gain can be achieved by modifying sensors, sctuators, or the controlled system itself. This should reduce the measurement error, eliminate the torque ripple, or raise the frequency of the unmodeled dynamics. Other approaches, like modeling flexibility, have the disadvantage that the model (1) can no longer be used, and that the number of inputs will be smaller than the number of degrees-of-freedom, making necessary the use of other, more-complicated, controllers.

The tracking error, which ideally should be zero, is caused by differences between the design model (2) and reality and can be attributed to differences between design and simulation model, and between simulation model and reality. The differences between design and simulation model (and between the ideal and actual implementation of the controller) are already discussed in Section 4. A comparison of the simulation and experimental results shows a difference in performance. This can be attributed to the erroneous model of the $\mathrm{XY}$-table. To be specific, the following discrepancies between simulation model and reality are known to exist

- the number of degrees-of-freedom of the model is too low, due to flexible connections, e.g., belts, torsion spring, connecting springs, that the model does not account for,

- actuator and sensor dynamics are not included in the model.

- backlash has been observed, but is not modeled,

- the model does not contain a specific low velocity friction term.

Compared with the controlled system bandwidth, the first unmodeled resonant mode for $x$ and $y$ direction is quite close, evident because increasing the feedback gains, and by that the bandwidth, readily caused unacceptable input signals, eventually leading to repeated instances of saturation and other extreme excursions of the control input.

The discrepancy between simulation and experiment means that an evaluation of modifications of control schemes by simulations should always be checked by an implementation of the modification in the controller software and validation of the simulation results with experiments. This indispensable step is, however. sometimes omitted in the development and presentation of control schemes. As also concluded by Berghuis (1993), the predictive value of simulations decreases when unmodeled dynamics becomes more dominant, which is increasingly the case if friction compensation is more involved.

\section{CONCLUSIONS AND RECOMMENDATIONS}

From the simulation results and the experiments it is concluded that the use of extended friction models can improve the tracking performance. Adaptation of the model parameters is necessary to get small tracking er- rors. The adaptation should be made fast to permit short adaptation times in case no previous knowledge of the parameters is available. In our setup this was not possible without influencing the stability. When previous knowledge of the parameters is available the allowable adaptation gains give a sufficiently fast parameter adaptation.

Further research in this area should focus on guidelines for the choice of the adaptation gain. The tuning rule proposed by Niemeyer and Slotine (1991) could not be used without additional adjustments of the gains. There is also a modest discrepancy between the simulation results and the experiments. To be able to evaluate modifications of control schemes with simulations only, a more accurate model must be made. To ease the interpretation of the results the authenticity of the model should be sufficient. All components of the friction model (4) were significant for the XY-table. Perhaps additional terms in this equation can still improve the tracking error.

\section{REFERENCES}

Armstrong, B. (1988). Friction: Experimental determination, modeling and compensation. In: Proc. of the 1988 IEEE Internat. Conf. on Robotics and Automat., Vol. 3.pp. 1422-1427.

Armstrong-Hélouvry, B. (1991). Control of machines with friction. Kluwer Academic Publishers, Boston.

Baril. C. G. and P.-O. Gutman (1991). Performance related adaptive friction compensation for uncertain systems. In: Proc. of the first European Control Conf., Vol. 1, pp. 248-251.

Berghuis, H. (1993). Model-based Robot Control: from Theory to Practice. PhD dissertation. University of Twente, Enschede, The Netherlands.

Bona, B. and M. Indri (1993). On the stability of force/position controlled manipulators in presence of friction and stiction. In: Preprints of the 12th IFAC World Congress. Vol. 7, pp. 411-416.

Brandenburg. G.. H. Hertle and K. Zeiselmair (1987). Dynamic influence and partial compensation of Coulomb friction in a position- and speed-controlled elastic two-mass system. In: Proc. of the 10th IFAC World Congress, Vol. 3, pp. 93-101.

Canudas, C., K. J. Áström and K. Braun (1987). Adaptive friction compensation in DC-motor drives, IEEE J. Robotics Automat., RA-3, 681-685.

Canudas de Wit. C.. P. Noël, A. Aubin and B. Brogliato (1991). Adaptive friction compensation in robot manipulators: Low velocities, Internat. J. Robotics Res., 10, 189-199.

de Jager, B. (1992). Robust control of mechanical systems: An experimental study. In: Proc. NOLCOS'92. (M. Fliess. Ed.). pp. 501-506.

Friedland. B. and Y.-J. Park (1992). On adaptive friction compensation, IEEE Trans. Automat. Control, 37, 1609-1612.

Gogoussis, A. and M. Donath (1988). Coulomb friction effects on the dynamics of bearings and transmissions in precision robot mechanisms. In: Proc. of the 1988 IEEE Internat. Conf. on Robotics and 
Automat., Vol. 3, pp. 1440-1446.

Hressig. Jr., D. A. and B. Friedland (1991). On the modeling and simulation of friction,J. Dynamic Systems, Measurement, and Control, 113, 354-362.

Hori, Y. and T. Uchida (1990). Proposal of a novel motion control method based on acceleration control, Electr. Engrg. Japan. 110, 67-76.

Johnson, C. T. and R. D. Lorenz (1992). Experimental identification of friction and its compensation in precise, position controlled mechanisms, IEEE Trans. Industry Applic., 28, 1392-1398.

Maron. J. C. (1989). Nonlinear identification and observer based compensation of friction in mechanical systems. In: Nonlinear Control Systems Design: science papers of the IFAC Symposium. pp. 397-402.

Niemeyer, G. and J.-J. E. Slotine (1991). Performance in adaptive manipulator control, Internat. J. Robotics Res., 10, 149-161.

Slotine, J.-J. E and W. Li (1988). Adaptive manipulator control: A case study, IEEE Trans. Automat. Control, AC-33, 995-1003.

Spong. M. W., R. Ortega and R. Kelly (1990). Comments on "Adaptive manipulator control: A case study", IEEE Trans. Automat. Control, 35, 761-762.

Tung, E. D., G. Anwar and M. Tomizuka (1993). Low velocity friction compensation and feedforward solution based on repetitive control. J. Dynamic Systems, Measurement, and Control, 115, 279-284.

van de Molengraft, M. J. G. (1990). Identification of nonlinear mechanical systems: for control applications. PhD dissertation. Eindhoven University of Technology, Eindhoven. The Netherlands.

Whitcomb, L. L., A. A. Rizzi and D. E. Koditschek (1993). Comparative experiments with a new adaptive controller for robot arms, IEEE Trans. Robotics Automat., 9. 59-70.

Yang, Y. P. and J. S. Chu (1993). Adaptive velocity control of DC motors with Coulomb friction identification. J. Dynamic Systems, Measurement, and Control, 115, 95-102.

\section{APPENDIX}

The model of the XY-table used in the simulations is detailed below in pseudo code.

$$
\begin{aligned}
& W:=a_{c}+b_{p} \sin \left(\omega_{p} z+\frac{\varphi_{p}^{+}+\varphi_{p}^{-}}{2}+\frac{\varphi_{p}^{+}-\varphi_{p}^{-}}{2} \operatorname{sgn} \dot{z}\right) \\
& V:=a_{\mathrm{v}} \dot{z} \\
& F:=\frac{u}{K}+2 a_{n}(\text { rand }-0.5) \\
& \text { if } z=0 \text { then } \\
& \quad \text { if }|F|>W \text { then } W:=W \operatorname{sgn} F \text { else } W:=F \\
& \text { else } W:=W \operatorname{sgn} \dot{z} \\
& z \ddot{z}:=\frac{F-W-V}{\vartheta_{1} \text { or } 2} \\
& \text { integrate (with detection of zero velocity) } \\
& \text { to obtain position } z \text { at next sampling instance } \\
& \text { pos }:=\text { round }\left(\frac{z-\text { off }}{\text { fac }}\right)
\end{aligned}
$$

The code for $x$ and $y$ direction is the same, only the parameters, as listed in Table 2, are different. Here $z$ is the $x$ or $y$ position [mm], $u$ the setpoint signal to the current amplifier [DA converter counts], and pos the measured output [encoder counts].

Due to the sampled data implementation of the controller, the input $u$ is constant during a sampling inter$\mathrm{val}$, and the periodic component of $W$ is assumed to be constant. This permits an easy analytic solution of the differential equation, so the integration is exact. The detection of zero velocity is for detecting changes in the sign of the friction force. Integration is performed over the subinterval up to $t(i=0)$, then the friction force $W$ is computed again, and the integration over the remaining part of the sampling interval is performed. It is possible for the system to stick during part of a sampling interval.

The function rand calls a random number generator with uniform distribution in the open interval $(0,1)$. Therefore, parameter $a_{n}$ is the maximum amplitude of a band-limited pseudo white noise disturbance force used to model torque ripple and other random disturbances. The value of $a_{n}$ is determined so the "roughness" of the simulation data is almost equal to the experimental data. No formal identification of this parameter has taken place. but the value is not unreasonable, being equivalent with $\sim 4 \%$ of the maximum motor torque. the same percentage as reported elsewhere (ArmstrongHélouvry, 1991; p. 44).

The function round rounds the function argument to the nearest integer and it models the quantization error of the encoder measurement.

The values for the viscous friction coefficients $a_{v}$ are by accident much higher than in practice. The values of $1 / \omega_{p}$ are slightly different from the radii of the belt

\begin{tabular}{|c|c|c|c|}
\hline Parameter & Value $x$ & Value $y$ & Unit \\
\hline$v_{1}, v_{2}$ & 46.5 & 4.3 & $\mathrm{~kg}$ \\
\hline$a_{c}^{+}=a_{c}^{-}$ & 45000 & 12500 & $\mathrm{mN}$ \\
\hline$a_{v}^{+}=a_{v}^{-}$ & 6.0 & 10.0 & $\mathrm{mN} s \mathrm{~mm}^{-1}$ \\
\hline$b_{p}^{+}=b_{p}^{-}$ & 12500 & 3500 & $\mathrm{mN}$ \\
\hline$\omega_{p}$ & $1 / 9.7$ & $1 / 10.5$ & $\operatorname{rad} \mathrm{mm}^{-1}$ \\
\hline$\varphi_{p}^{+} / \omega_{p}$ & -815 & -790 & $\mathrm{~mm}$ \\
\hline$\varphi_{p}^{-} / \omega_{p}$ & -835 & -820 & $\mathrm{~mm}$ \\
\hline$a_{n}$ & 6250 & 2500 & $\mathrm{mN}$ \\
\hline$K$ & .0080605 & .0318878 & counts $\mathrm{mN}^{-1}$ \\
\hline off & 280 & 1170 & $\mathrm{~mm}$ \\
\hline fac & .0035316 & .0162999 & $\mathrm{~mm}$ counts $^{-1}$ \\
\hline
\end{tabular}
wheels. to test the ability of adapting a phase shift $\Phi_{p}$ that effectively changes in time. The other parameters are taken from identification experiments (van de Molengraft, 1990) or computed from data sheets of the manufacturer and are believed to agree reasonable well with reality, except for the Coulomb friction parameter that changes with time and whose determination is not very repeatable.

Table 2 Simulation model parameters 